\title{
Effects of Radiation on Unsteady Couette Flow between Two Vertical Parallel Plates with Ramped Wall Temperature
}

\author{
S. Das \\ Department of Mathematics, \\ University of Gour Banga, \\ Malda 732 103, India
}

\author{
C. Mandal \\ Department of Applied \\ Mathematics, Vidyasagar \\ University, Midnapore 721 102, \\ India
}

\author{
R. N. Jana \\ Department of Applied \\ Mathematics, Vidyasagar \\ University, Midnapore 721 102, \\ India
}

\begin{abstract}
The effect of radiation on unsteady Couette flow between two vertical parallel plates with ramped wall temperature have been studied. The fluid considered here is a gray, absorbing/emitting radiation but a non-scattering medium. An analytical solution of the governing equations has been obtained by employing Laplace transform technique. The influence of the various parameters, entering into the problem, on the velocity field, temperature field, skin friction and rate of heat transfer is discussed with the help of graphs and tables. It is found that an increase in radiation parameter leads to decrease the fluid velocity and temperature. It is also found that both the velocity as well as the temperature of the fluid decrease with an increase in Prandtl number. Further it is observed that the velocity increases with an increase in Grashof number and an increase in time leads to increase the fluid velocity and temperature.
\end{abstract}

Keywords: Couette flow, Radiation parameter, Prandtl number, Grashof number and Ramped wall temperature.

\section{INTRODUCTION}

An analysis of flow formation in Couette motion, as predicted by classical fluid mechanics, was presented by Schlichting [1] This problem is of fundamental importance as it provides the exact solution of the Navier-Stokes equation and reveals how the velocity profiles varies with time, approaching a linear distribution asymptotically, and how the boundary layer spreads throughout the flow field. Couette flow is one of the basic flow in fluid dynamics that refers to the laminar flow of a viscous fluid in the space between two parallel plates, one of which is moving relative to the other. The flow is driven by virtue of viscous drag force acting on the fluid and the applied pressure gradient parallel to the plates. Couette flow is frequently used in physics and engineering to illustrate shear-driven fluid motion. The radiative heat transfer as an important fundamental phenomena existing in practical engineering such as those found in solar radiation in buildings, foundry engineering and solidification processes, die forging, chemical engineering, composite structures applied in industry Korycki[2]. Another important feature that usually occurs in electronic devices over a period of continuous usage is the hotness of the surface. This means that a poor design could trap heat generated by the source of the power supply and could incapacitate the efficiency and durability of the systems. Therefore, the efficiency in the functioning of these systems is enhanced when they are subjected to external cooling devices like air conditioners, electric fans, and some others (e.g. laptop, computers) inbuilt storage devices that store electrical energy for them to function for sometime even without external source of power supply Gbaorun et al.[3]. The IC components of these electronic systems are thermally coupled to the surrounding via convection and radiation. Radiation has a significant role in heat transfer in low-flow applications where there exists a larger temperature gradient between the components and the surrounding. Radiative free convection Couette flows are frequently encountered in many scientific and environmental processes, such as heating and cooling of chambers and solar power technology. Heat transfer by simultaneous radiation and convection has applications in numerous technological problems including combustion, furnace design, the design of high temperature gas cooled nuclear reactors, nuclear reactor safety, fluidized bed heat exchanger, fire spreads, solar fans, solar collectors natural convection in cavities, turbid water bodies, photo chemical reactors and many others. Further, the heat transfer effects under the conditions of free convection are now dominant in many engineering applications such as rocket nozzels, high sinks in turbine blades, high speed aircrafts and their atmospheric re-entry, chemical devices and process equipment, formation and dispersion of fog, distribution of temperature and moisture over agricultural field and groves of fruit trees, damage of crops due to freezing and pollution of the environment and so on. Singh [4] has studied the natural convection in unsteady Couette motion. Ogulu and Motsa [5] have investigated the radiative heat transfer to magnetohydrodynamic Couette flow with variable wall temperature. The radiation effects on MHD Couette flow with heat transfer between two parallel plates have been describbed by Mebine [6]. Effects of thermal radiation and free convection currents on the unsteady Couette flow between two vertical parallel plates with constant heat flux at one boundary have been examined by Narahari [7]. Deka and Bhattacharya [8] have analyzed the unsteady free convective Couette flow of heat generating/absorbing fluid in porous medium. The radiation effect on electrohydrodynamic froth flow in vertical channel have been examined by Gbadeyan et al. [9].

The present work concerns with the effect of radiation on unsteady Coutte flow of a viscous incompressible fluid confined between two vertical parallel plates with ramped wall temperature. Initially, at time $t \leq 0$, both the fluid and plate are at rest and constant temperature $T_{h}$. An exact solution of the governing equations have been obtained by using Laplace transformation technique. It is found that the velocity decreases with an increase in either radiation parameter $R$ or Prandtl number $\mathrm{Pr}$. An increase in Grashof number $\mathrm{Gr}$ leads to rise the velocity $u_{1}$ of flow. It is seen that the velocity $u_{1}$ increases with an increase in time $\tau$. It is also seen that the temperature $\theta$ decreases with an increase in either radiation parameter $R$ 
or Prandtl number $\operatorname{Pr}$ but it increases with an increase in time $\tau$. The shear stress $\tau_{x}$ decreases for both the cases of ramped and isothermal wall temperature with an increase in either radiation parameter $R$ or Prandtl number $\mathrm{Pr}$ for fixed values of Grashof number $\mathrm{Gr}$. Further, the rate of heat transfer $-\theta^{\prime}(0)$ decreases with an increase in either radiation parameter $R$ or Prandtl number $P r$ for both ramped as well as isothermal wall temperature at the wall $\eta=0$.

\section{FORMULATION OF THE PROBLEM AND ITS SOLUTIONS}

Consider the unsteady Couette flow of a viscous incompressible radiative heat generating fluid between two infinite vertical parallel walls separated by a distance $h$. The flow is set up by the buoyancy force arising from the temperature gradient occurring as a result of asymmetric heating of the parallel plates as well as constant motion of one of the plates. Choose a cartesian co-ordinates system with the $x$-axis along one of the plates in the vertically upward direction and the $y$-axis normal to the plates [See Figure 1]. Initially, at time $t \leq 0$, the two plates and the fluid are assumed to be at the same temperature $T_{h}$ and stationary. At time $t>0$, the plate at $y=0$ starts moving in its own plane with a velocity $\lambda^{*} U(t)$ and the temperature of the plate is raised or lowered to $T_{h}+\left(T_{0}-T_{h}\right) \frac{t}{t_{0}}$ when $0<t \leq t_{0}$ and the constant temperature $T_{h}$ is maintained at $t>t_{0}$ whereas the plate at $y=h$ is stationary and maintained at a constant temperature $T_{h}$, where $\lambda^{*}$ is a constant. Our aim is to analyze the radiation effects on the unsteady Couette flow resulting from the ramped temperature profile of the moving plate. We assume that the flow is laminar and is such that the effects of the convective and pressure gradient terms in the momentum and energy equations can be neglected. It is assumed that the effect of viscous dissipation is negligible. It is also assumed that the radiative heat flux in the $x$ - direction is negligible as compared to that in the $y$ direction. As the plates are infinite long, the velocity and temperature fields are functions of $y$ and $t$ only.

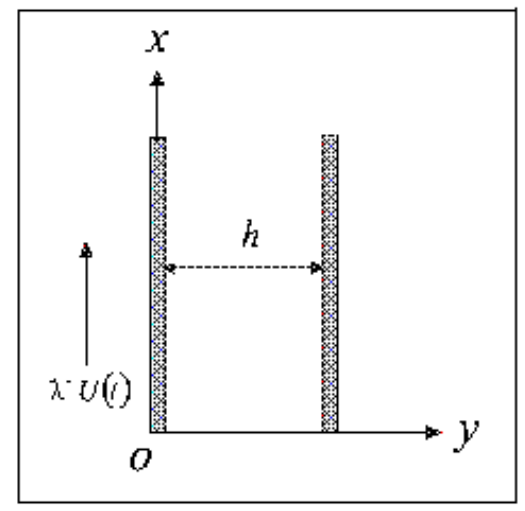

Figure 1. Geometry of the problem

The free convection flow of a radiating fluid, under usual Boussinesq approximation, to be governed by the following system of equations

$$
\begin{aligned}
& \frac{\partial u}{\partial t}=g \beta\left(T-T_{h}\right)+v \frac{\partial^{2} u}{\partial y^{2}}, \\
& \rho c_{p} \frac{\partial T}{\partial t}=k \frac{\partial^{2} T}{\partial y^{2}}-\frac{\partial q_{r}}{\partial y},
\end{aligned}
$$

where $u$ is the velocity in the $x$-direction, $T$ the temperature of the fluid, $g$ the acceleration due to gravity, $v$ the kinematic coefficient of viscosity, $\rho$ the fluid density, $k$ the thermal conductivity, $c_{p}$ the specific heat at constant pressure and $q_{r}$ the radiative heat flux. The heating due to viscous dissipation is neglected for small velocities in the energy equation (2).

The initial and the boundary conditions are

$$
\begin{aligned}
& u=0, T=T_{h} \text { for } 0 \leq y \leq h \text { and } t \leq 0, \\
& u=\lambda^{*} U(t) \text { at } y=0, \\
& T=\left\{\begin{array}{ll}
T_{h}+\left(T_{0}-T_{h}\right) \frac{t}{t_{0}} & \text { for } 0<t \leq t_{0} \\
T_{h} & \text { for } t>t_{0}
\end{array} \text { at } y=0\right. \\
& u=0, T=T_{h} \text { at } y=h \text { for } t>0
\end{aligned}
$$

The radiative heat flux can be found from Rosseland approximation and its formula is derived from the diffusion concept of radiative heat transfer in the following way

$$
q_{r}=-\frac{4 \sigma}{3 k^{*}} \frac{\partial T^{4}}{\partial y},
$$

where $\sigma$ is the Stefan-Boltzman constant and $k^{*}$ the spectral mean absorption coefficient of the medium. It should be noted that by using the Rosseland approximation we limit our analysis to optically thick fluids. If the temperature differences within the flow are sufficiently small, then the equation (4) can be linearized by expanding $T^{4}$ into the Taylor series about $T_{h}$ and neglecting higher order terms to give:

$$
T^{4}=4 T_{h}^{3} T-3 T_{h}^{4} \text {. }
$$

It is emphasized here that equation (5) is widely used in computational fluid dynamics involving radiation absorption problems [10] in expressing the term $T^{4}$ as a linear function. In view of (4) and (5), equation (2) reduces

$$
\rho c_{p} \frac{\partial T}{\partial t}=k \frac{\partial^{2} T}{\partial y^{2}}+\frac{16 \sigma T_{h}^{3}}{3 k^{*}} \frac{\partial^{2} T}{\partial y^{2}} .
$$

Introducing non-dimensionless variables

$$
\begin{aligned}
& \eta=\frac{y}{h}, \tau=\frac{t}{t_{0}}, t_{0}=\frac{h^{2}}{v}, u_{1}=\frac{u}{U_{0}}, \\
& \theta=\frac{T-T_{h}}{T_{0}-T_{h}}, U(t)=U_{0} f(\tau),
\end{aligned}
$$

equations (1) and (6) become

$$
\begin{aligned}
& \frac{\partial u_{1}}{\partial \tau}=\frac{\partial^{2} u_{1}}{\partial \eta^{2}}+G r \theta, \\
& \alpha \frac{\partial \theta}{\partial \tau}=\frac{\partial^{2} \theta}{\partial \eta^{2}},
\end{aligned}
$$

where $\alpha=\frac{3 R P r}{3 R+4}$ and $R=\frac{k k^{*}}{4 \sigma T_{h}^{3}}$ is the radiation parameter, $\operatorname{Pr}=\frac{\rho v c_{p}}{k}$ the Prandtl number and $G r=\frac{g \beta\left(T_{0}-T_{h}\right) h^{2}}{v U_{0}}$ the Grashof number. 
The corresponding initial and the boundary conditions are $u_{1}=0, \theta=0$ for $0 \leq \eta \leq 1$ and $\tau \leq 0$,

$$
\begin{aligned}
& u_{1}=\lambda f(\tau), \theta=\left\{\begin{array}{ll}
\tau & \text { for } 0<\tau \leq 1 \\
1 & \text { for } \tau>1
\end{array} \text { at } \eta=0\right. \\
& u_{1}=0, \theta=0 \text { at } \eta=1 \text { for } \tau>0,
\end{aligned}
$$

where $\lambda=\frac{\lambda^{*} v}{U_{0}^{2}}$ is the accelerating parameter.

On the use of Laplace transformation, equations (8) and (9) become

$$
s \bar{u}_{1}=\frac{d^{2} \bar{u}_{1}}{d \eta^{2}}+G r \bar{\theta},
$$

$$
\begin{aligned}
& \bar{\theta}(\eta, s)= \begin{cases}\frac{\left(1-e^{-s}\right)}{s^{2}} \sum_{n=0}^{\infty}\left[e^{-a \sqrt{\alpha s}}-e^{-b \sqrt{\alpha s}}\right] & \text { for } \alpha \neq 1, \\
\frac{\left(1-e^{-s}\right)}{s^{2}} \sum_{n=0}^{\infty}\left[e^{-a \sqrt{s}}-e^{-b \sqrt{s}}\right] & \text { for } \alpha=1,\end{cases} \\
& \bar{u}_{1}(\eta, s)= \begin{cases}\lambda \bar{f}(s) \sum_{n=0}^{\infty}\left[e^{-a \sqrt{s}}-e^{-b \sqrt{s}}\right] & \text { for } \alpha \neq 1, \\
+\frac{G r\left(1-e^{-s}\right)}{s^{3}(\alpha-1)} \sum_{n=0}^{\infty}\left[\left(e^{-a \sqrt{s}}-e^{-b \sqrt{s}}\right)-\left(e^{-a \sqrt{\alpha s}}-e^{-b \sqrt{\alpha s}}\right)\right] & \\
\lambda \bar{f}(s) \sum_{n=0}^{\infty}\left[e^{-a \sqrt{s}}-e^{-b \sqrt{s}}\right]+\frac{G r\left(1-e^{-s}\right)}{2 s^{5 / 2}}\left[\eta \sum_{n=0}^{\infty}\left(e^{-a \sqrt{s}}-e^{-b \sqrt{s}}\right)\right. & \text { for } \alpha=1 . \\
-2 \sum_{n=0}^{\infty}(n+1)\left\{e^{-b \sqrt{s}}-e^{-d \sqrt{s}}\right\} & \end{cases}
\end{aligned}
$$

where

$$
\bar{u}_{1}(\eta, s)=\int_{0}^{\infty} u_{1}(\eta, s) e^{-s \tau} d \tau, \bar{\theta}(\eta, s)=\int_{0}^{\infty} \theta(\eta, s) e^{-s \tau} d \tau
$$

The corresponding boundary conditions for $\bar{u}_{1}$ and $\bar{\theta}$ are

$$
\begin{aligned}
& \bar{u}_{1}(0, s)=\lambda \bar{f}(s), \bar{u}_{1}(1, s)=0, \\
& \bar{\theta}(0, s)=\frac{1}{s^{2}}\left(1-e^{-s}\right), \quad \bar{\theta}(1, s)=0 .
\end{aligned}
$$

The solution of the equations (11) and (12) subject to the boundary conditions (14) are easily obtained and are given by

The inverse transforms of (15) and (16) give the solution for the temperature distribution and velocity field as

$$
\theta(\eta, \tau)= \begin{cases}\sum_{n=0}^{\infty}\left[F_{2}(a \sqrt{\alpha}, \tau)-F_{2}(b \sqrt{\alpha}, \tau)\right. & \text { for } \alpha \neq 1, \\ \left.-H(\tau-1)\left\{F_{2}(a \sqrt{\alpha}, \tau-1)-F_{2}(b \sqrt{\alpha}, \tau-1)\right\}\right] & \\ \sum_{n=0}^{\infty}\left[F_{2}(a, \tau)-F_{2}(b, \tau)-H(\tau-1)\left\{F_{2}(a, \tau-1)-F_{2}(b, \tau-1)\right\}\right] & \text { for } \alpha=1,\end{cases}
$$




$$
u_{1}(\eta, \tau)= \begin{cases}\lambda \sum_{n=0}^{\infty}[F(a, \tau)-F(b, \tau)]+\frac{G r}{\alpha-1} \sum_{n=0}^{\infty}\left[F_{1}(a, \tau)-F_{1}(b, \tau)\right. & \\ -\left\{F_{1}(a \sqrt{\alpha}, \tau)-F_{1}(b \sqrt{\alpha}, \tau)\right\} & \\ -H(\tau-1)\left\{F_{1}(a, \tau-1)-F_{1}(b, \tau-1)\right. & \\ \left.\left.-\left[F_{1}(a \sqrt{\alpha}, \tau-1)-F_{1}(b \sqrt{\alpha}, \tau-1)\right]\right\}\right] & \text { for } \alpha \neq 1, \\ \lambda \sum_{n=0}^{\infty}[F(a, \tau)-F(b, \tau)]+\frac{G r}{2} \sum_{n=0}^{\infty} \eta\left[F_{3}(a, \tau)-F_{3}(b, \tau)\right. & \\ \left.-H(\tau-1)\left\{F_{3}(a, \tau-1)-F_{3}(b, \tau-1)\right\}\right] & \\ -G r \sum_{n=0}^{\infty}(n+1)\left[F_{3}(b, \tau)-F_{3}(d, \tau)\right. & \\ \left.-H(\tau-1)\left\{F_{3}(b, \tau-1)-F_{3}(d, \tau-1)\right\}\right] & \text { for } \alpha=1,\end{cases}
$$

where

$$
\begin{aligned}
& a=2 n+\eta, b=2 n+2-\eta, d=2 n+2+\eta \\
& F(z, \tau)=\operatorname{erfc}\left(\frac{z}{2 \sqrt{\tau}}\right) \text {, } \\
& F_{1}(z, \tau)=\frac{1}{2}\left(\tau^{2}+\tau z^{2}+\frac{1}{12} z^{4}\right) \operatorname{erfc}\left(\frac{z}{2 \sqrt{\tau}}\right) \\
& -\frac{1}{6} z \sqrt{\frac{\tau}{\pi}}\left(5 \tau+\frac{1}{2} z^{2}\right) e^{-\frac{z^{2}}{4 \tau}}, \\
& F_{2}(z, \tau)=\left(\tau+\frac{z^{2}}{2}\right) \operatorname{erfc}\left(\frac{z}{2 \sqrt{\tau}}\right)-z \sqrt{\frac{\tau}{\pi}} e^{-\frac{z^{2}}{4 \tau}}, \\
& F_{3}(z, \tau)=\frac{1}{3} \sqrt{\frac{\tau}{\pi}}\left(4 \tau+z^{2}\right) e^{-\frac{z^{2}}{4 \tau}}-z\left(\tau+\frac{z^{2}}{6}\right) \operatorname{erfc}\left(\frac{z}{2 \sqrt{\tau}}\right)
\end{aligned}
$$

and $z$ is a dummy variable and $F_{1}, F_{2}$ and $F_{3}$ are dummy functions defined in equation (19), $\operatorname{erfc}(x)$ being complementary error function and and $H(\tau-1)$ is the unit step function. It is noticed that in the absence of radiation parameter $(R=\infty)$, the solutions (17) and (18) are identical with the equations (8) and (9).

\subsection{SOLUTION FOR ISOTHERMAL PLATES}

For isothermal plates, the boundary conditions for the temperature and velocity fields are

$$
\bar{u}_{1}(\eta, s)= \begin{cases}\lambda \bar{f}(s) \sum_{n=0}^{\infty}\left[e^{-a \sqrt{s}}-e^{-b \sqrt{s}}\right] \\ +\frac{G r}{s^{2}(\alpha-1)} \sum_{n=0}^{\infty}\left[\left(e^{-a \sqrt{s}}-e^{-b \sqrt{s}}\right)-\left(e^{-a \sqrt{\alpha s}}-e^{-b \sqrt{\alpha s}}\right)\right] & \text { for } \alpha \neq 1, \\ \lambda \bar{f}(s) \sum_{n=0}^{\infty}\left[e^{-a \sqrt{s}}-e^{-b \sqrt{s}}\right]+\frac{G r}{2 s^{3 / 2}}\left[\eta \sum_{n=0}^{\infty}\left(e^{-a \sqrt{s}}-e^{-b \sqrt{s}}\right)\right. & \\ -2 \sum_{n=0}^{\infty}(n+1)\left\{e^{-b \sqrt{s}}-e^{-d \sqrt{s}}\right\} & \text { for } \alpha=1 .\end{cases}
$$

The inverse transforms of (23) and (24) give the solution for the temperature distribution and velocity field as 


$$
\begin{aligned}
\theta(\eta, \tau)= \begin{cases}\sum_{n=0}^{\infty}[F(a \sqrt{\alpha}, \tau)-F(b \sqrt{\alpha}, \tau)] & \text { for } \alpha \neq 1, \\
\sum_{n=0}^{\infty}[F(a, \tau)-F(b, \tau)] \quad \text { for } \alpha=1,\end{cases} \\
u_{1}(\eta, \tau)=\left\{\begin{array}{ll}
\lambda \sum_{n=0}^{\infty}[F(a, \tau)-F(b, \tau)] \\
+\frac{G r}{\alpha-1} \sum_{n=0}^{\infty}\left[F_{2}(a, \tau)-F_{2}(b, \tau)-\left\{F_{2}(a \sqrt{\alpha}, \tau)-F_{2}(b \sqrt{\alpha}, \tau)\right\}\right] \\
\lambda \sum_{n=0}^{\infty}[F(a, \tau)-F(b, \tau)]+\frac{G r}{2} \sum_{n=0}^{\infty} \eta\left[F_{4}(a, \tau)-F_{4}(b, \tau)\right] \\
-2 \sum_{n=0}^{\infty}(n+1)\left[F_{4}(b, \tau)-F_{4}(d, \tau)\right]
\end{array} \quad \text { for } \alpha \neq 1,\right.
\end{aligned}
$$

where $a, b, d$ and $F(z, \tau)$ are given by (19) and

$$
F_{4}(z, \tau)=2 \sqrt{\frac{\tau}{\pi}} e^{-\frac{z^{2}}{4 \tau}}-z \operatorname{erfc}\left(\frac{z}{2 \sqrt{\tau}}\right) .
$$

\section{RESULTS AND DISCUSSION}

We have presented the non-dimensional velocity $u_{1}$ and temperature $\theta$ for several values of Radiation parameter $R$, Prandtl number $P r$, Grashof number $G r$ and time $\tau$ in Figures 2-8. It is found from Figures 2 and 3 that the velocity $u_{1}$ decreases with an increase in either radiation parameter $R$ or Prandtl number $P r$. This is possible physically because fluids with high Prandtl number have greater viscosity, which makes the fluid thick and hence move slowly. It is also found that the velocity $u_{1}$ is maximum near the plate and decreases away from the plate and finally takes asymptotic value for all values of $R$ and $P r$. It is observed from Figures 4 and 5 that an increase in either Grashof number $G r$ or time $\tau$ leads to rise in the velocity $u_{1}$. Physically this is due to fact that as the Grashof number $G r$ or time $\tau$ increases, the contribution from the buoyancy force near the plate becomes significant and hence a rise in the velocity near the plate as observed. It is observed from Figures 6 and 7 that the temperature $\theta$ decreases with an increase in either radiation parameter $R$ or Prandtl number $\operatorname{Pr}$. In the presence of radiation, the thermal boundary layer always found to thicken which implies that the radiation provides an additional means to diffuse energy. This means that the thermal boundary layer decreases and more uniform temperature distribution across the boundary layer. An increase in the Prandtl number means an increase of fluid viscosity, which causes a decrease in the flow velocity and the temperature decreases. This is consistent with the fact that the thermal boundary layer thickness decreases with the increasing Prandtl number. The temperature $\theta$ increases with an increase in time $\tau$ shown in Figure 8

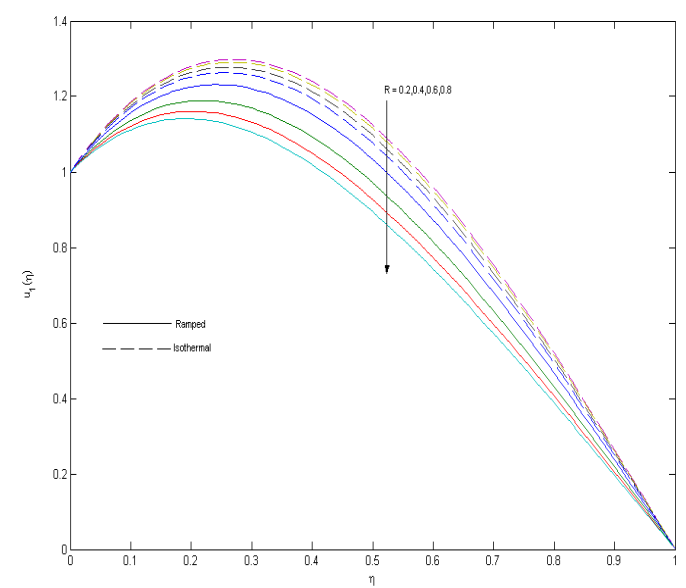

Figure 2. Velocity profiles for $R$ when $P r=10, G r=10$ and $\tau=\mathbf{1 . 1}$

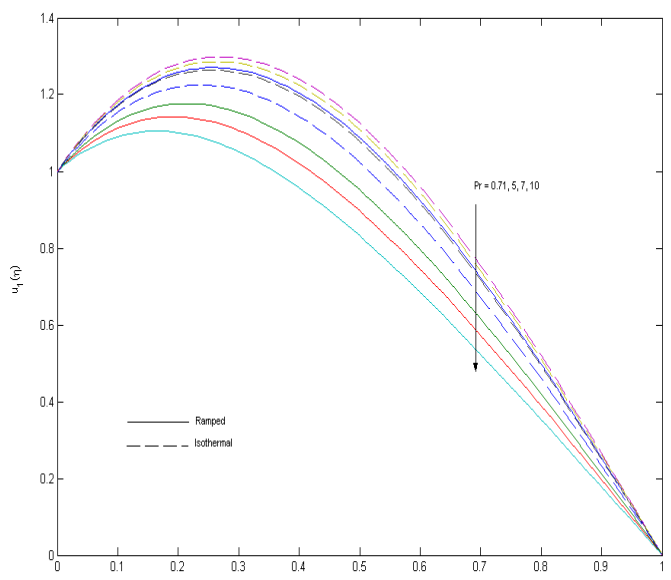

Figure 3. Velocity profiles for $\operatorname{Pr}$ when $R=1.5, G r=10$ and $\tau=1.1$ 


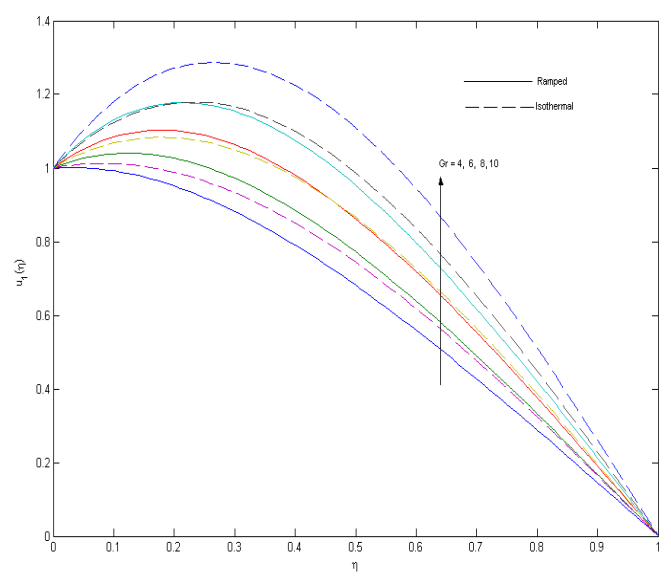

Figure 4. Velocity profiles for $G r$ when $P r=7, R=0.8$ and $\tau=1.1$

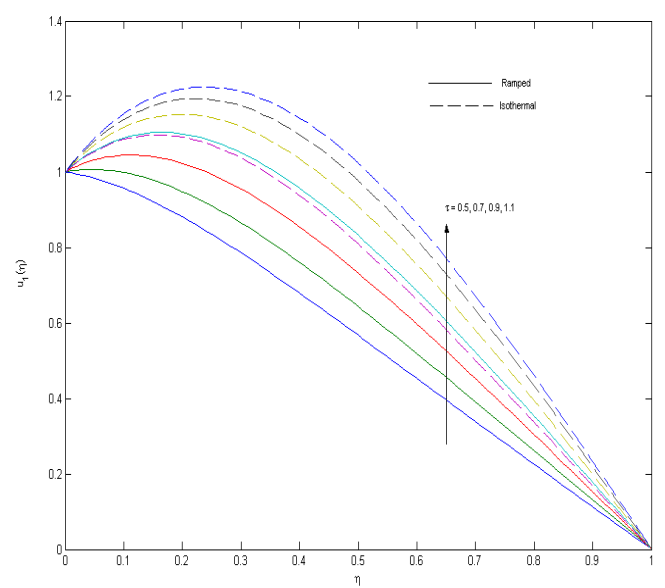

Figure 5. Velocity profiles for time $\tau$ when $\operatorname{Pr}=10$, $G r=10$ and $R=1.5$

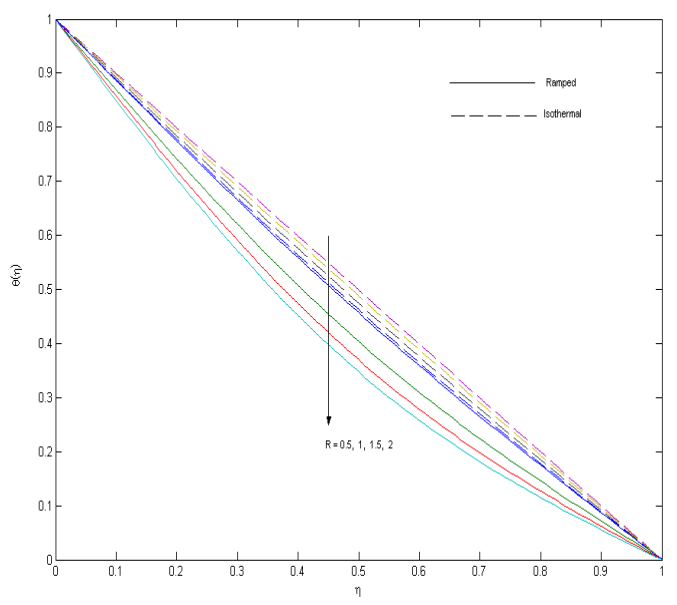

Figure 6. Temperature profiles for $R$ when $P r=7$ and $\tau=\mathbf{1 . 2}$

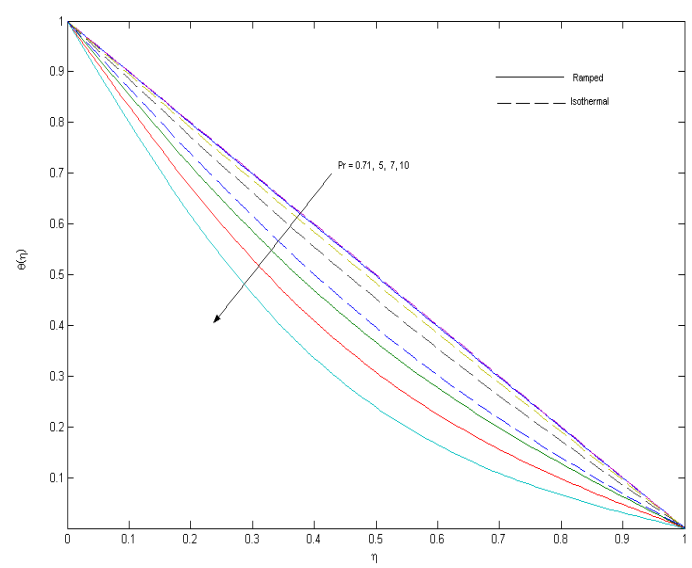

Figure 7. Temperature profiles for $\operatorname{Pr}$ when $R=2$ and $\tau=\mathbf{1 . 2}$

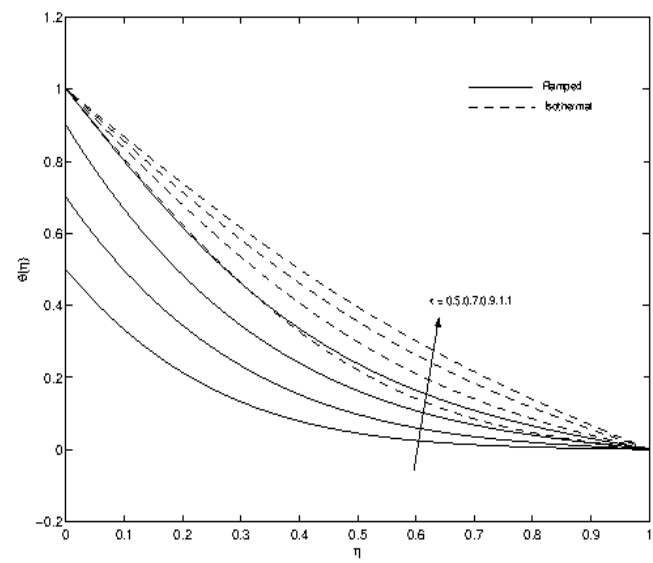

Figure 8. Temperature profiles for time $\tau$ when $\operatorname{Pr}=10$ and $R=2$

For ramped wall temperature, the shear stress at the plate $\eta=0$ is given by

$$
\tau_{x}=\left\{\begin{array}{l}
-\lambda \sum_{n=0}^{\infty}\left[H_{1}\left(a^{\prime}, \tau\right)-H_{1}\left(b^{\prime}, \tau\right)\right] \\
+\frac{G r}{\alpha-1} \sum_{n=0}^{\infty}\left[H_{2}\left(a^{\prime}, \tau\right)+H_{2}\left(b^{\prime}, \tau\right)\right. \\
-\sqrt{\alpha}\left\{H_{2}\left(a^{\prime} \sqrt{\alpha}, \tau\right)+H_{2}\left(b^{\prime} \sqrt{\alpha}, \tau\right)\right\} \\
-H(\tau-1)\left\{H_{2}\left(a^{\prime}, \tau-1\right)+H_{2}\left(b^{\prime}, \tau-1\right)\right. \\
\left.\left.-\sqrt{\alpha}\left[H_{2}\left(a^{\prime} \sqrt{\alpha}, \tau-1\right)+H_{2}\left(b^{\prime} \sqrt{\alpha}, \tau-1\right)\right]\right\}\right] \quad \text { for } \alpha \neq 1, \\
-\lambda \sum_{n=0}^{\infty}\left[H_{1}\left(a^{\prime}, \tau\right)-H_{1}\left(b^{\prime}, \tau\right)\right] \\
+\frac{G r}{2} \sum_{n=0}^{\infty}\left[F_{3}\left(a^{\prime}, \tau\right)-F_{3}\left(b^{\prime}, \tau\right)\right. \\
+2(n+1)\left\{H_{3}\left(b^{\prime}, \tau\right)+H_{3}\left(d^{\prime}, \tau\right)\right\} \\
-H(\tau-1)\left\{F_{3}\left(a^{\prime}, \tau-1\right)-F_{3}\left(b^{\prime}, \tau-1\right)\right. \\
\left.\left.+2(n+1)\left\{H_{3}\left(b^{\prime}, \tau-1\right)+H_{3}\left(d^{\prime}, \tau-1\right)\right\}\right\}\right] \quad \text { for } \alpha=1
\end{array}\right.
$$


where

$$
\begin{gathered}
a^{\prime}=2 n, b^{\prime}=2 n+2, \\
H_{1}(z, \tau)=-\frac{1}{\sqrt{\tau \pi}} e^{-\frac{z^{2}}{4 \tau}}, \\
H_{2}(z, \tau)=\left(z \tau+\frac{1}{6} z^{3}\right) \operatorname{erfc}\left(\frac{z}{2 \sqrt{\tau}}\right) \\
-\frac{1}{3} \sqrt{\frac{\tau}{\pi}}\left(4 \tau+z^{2}\right) e^{-\frac{z^{2}}{4 \tau}}, \\
H_{3}(z, \tau)=z \sqrt{\frac{\tau}{\pi}} e^{-\frac{z^{2}}{4 \tau}}-\left(\tau+\frac{z^{2}}{2}\right) \operatorname{erfc}\left(\frac{z}{2 \sqrt{\tau}}\right) .
\end{gathered}
$$

For isothermal plates, the shear stress at the plate $\eta=0$ is obtained as:

$$
\tau_{x}=\left\{\begin{array}{l}
-\lambda \sum_{n=0}^{\infty}\left[H_{1}\left(a^{\prime}, \tau\right)-H_{1}\left(b^{\prime}, \tau\right)\right] \\
+\frac{G r}{\alpha-1} \sum_{n=0}^{\infty}\left[H_{5}\left(a^{\prime}, \tau\right)+H_{5}\left(b^{\prime}, \tau\right)\right. \\
\left.-\sqrt{\alpha}\left\{H_{5}\left(a^{\prime} \sqrt{\alpha}, \tau\right)+H_{5}\left(b^{\prime} \sqrt{\alpha}, \tau\right)\right\}\right] \quad \text { for } \alpha \neq 1, \\
-\lambda \sum_{n=0}^{\infty}\left[H_{1}\left(a^{\prime}, \tau\right)-H_{1}\left(b^{\prime}, \tau\right)\right] \\
+\frac{G r}{2} \sum_{n=0}^{\infty}\left[F_{4}\left(a^{\prime}, \tau\right)-F_{4}\left(b^{\prime}, \tau\right)\right. \\
\left.+2(n+1)\left\{H_{4}\left(b^{\prime}, \tau\right)+H_{4}\left(b^{\prime}, \tau\right)\right\}\right] \quad \text { for } \alpha=1,
\end{array}\right.
$$
where

$$
\begin{aligned}
& H_{4}(z, \tau)=-\operatorname{erfc}\left(\frac{z}{2 \sqrt{\tau}}\right), \\
& H_{5}(z, \tau)=z \operatorname{erfc}\left(\frac{z}{2 \sqrt{\tau}}\right)-2 \sqrt{\frac{\tau}{\pi}} e^{-\frac{z^{2}}{4 \tau}} .
\end{aligned}
$$

and $a^{\prime}$ and $b^{\prime}$ and $H_{1}(z, \tau)$ are given by (29) and (30).

Numerical values of the non-dimensional shear stress at the moving plate $\eta=0$ are presented in Figures 9-11 against Grashof number $\mathrm{Gr}$ for various values of radiation parameter $R$, Prandtl number $P r$ and time $\tau$. Figure 9 shows that the shear stress $\tau_{x}$ at the moving plate $\eta=0$ increases for both ramped as well as isothermal wall temperature with an increase in either Grashof number $G r$ or time $\tau$. It is seen from Figures 10 and 11 that for fixed values of Grashof number $G r$, the shear stress $\tau_{x}$ decreases for both the cases of ramped and isothermal wall temperature with an increase in either radiation parameter $R$ or time $\tau$.

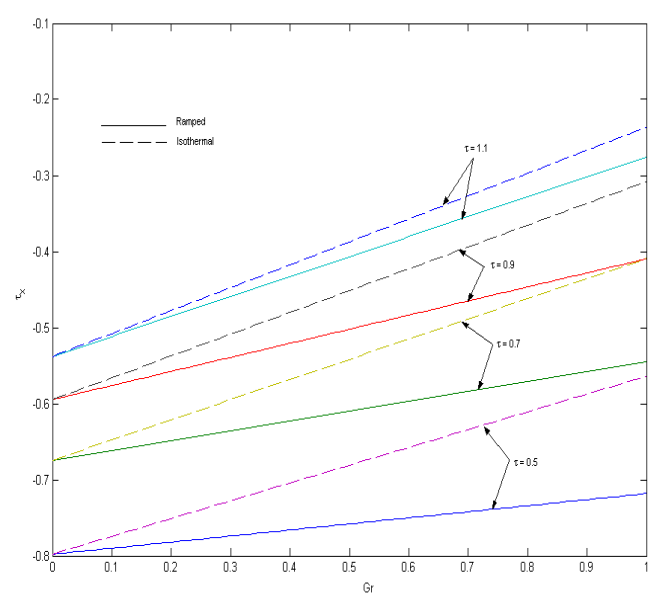

Figure 9. Shear stress $\tau_{x}$ for time $\tau$ when $\operatorname{Pr}=10$ and $R=1.5$

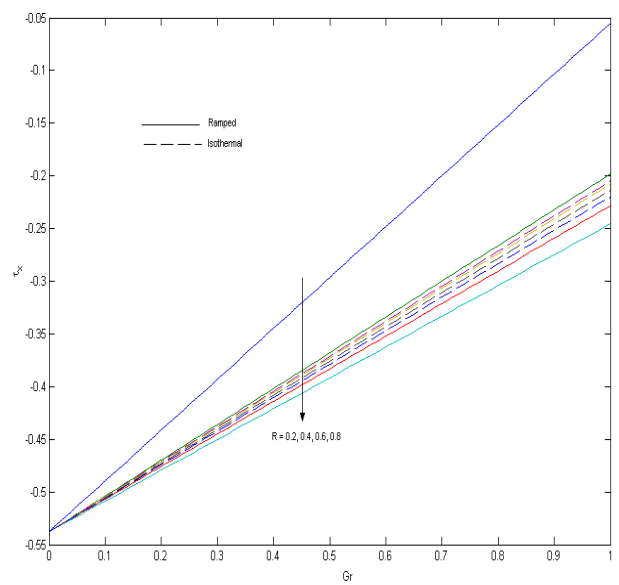

Figure 10. Shear stress $\tau_{x}$ for $R$ when $\operatorname{Pr}=10$ and $\tau=1.1$.

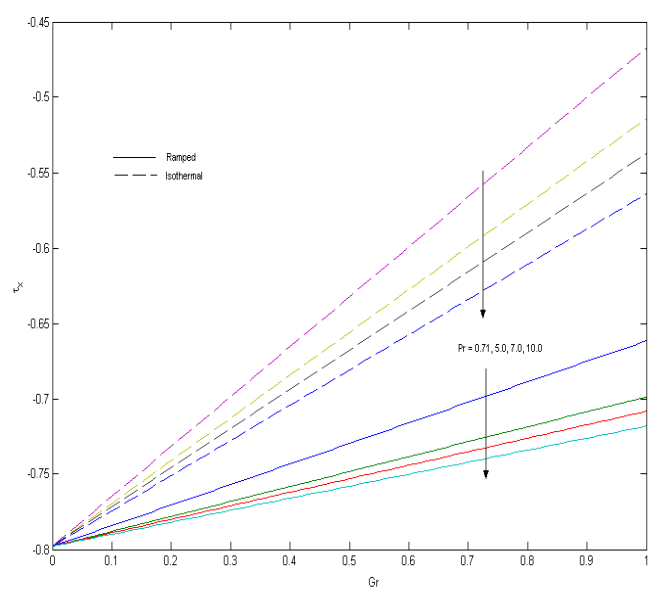

Figure 11. Shear stress $\tau_{x}$ for $\operatorname{Pr}$ when $\tau=0.5$ and $R=1.5$

For ramped wall temperature, the rate of heat transfer at the moving plate $\eta=0$ is obtained as: 


$$
\left.\theta^{\prime}(0)=\frac{\partial \theta}{\partial \eta}\right)_{\eta=0}= \begin{cases}\sum_{n=0}^{\infty}\left[\sqrt{\alpha}\left\{H_{6}\left(a^{\prime} \sqrt{\alpha}, \tau\right)+H_{6}\left(b^{\prime} \sqrt{\alpha}, \tau\right)\right\}\right. & \\ \left.-\sqrt{\alpha} H(\tau-1)\left\{H_{6}\left(a^{\prime} \sqrt{\alpha}, \tau-1\right)+H_{6}\left(b^{\prime} \sqrt{\alpha}, \tau-1\right)\right\}\right] & \text { for } \alpha \neq 1, \\ \sum_{n=0}^{\infty}\left[\left\{H_{6}\left(a^{\prime}, \tau\right)+H_{6}\left(b^{\prime}, \tau\right)\right\}\right. & \text { for } \alpha=1, \\ \left.-H(\tau-1)\left\{H_{6}\left(a^{\prime}, \tau-1\right)+H_{6}\left(b^{\prime}, \tau-1\right)\right\}\right] & \end{cases}
$$

where

$$
H_{6}(z, \tau)=z \operatorname{erfc}\left(\frac{z}{2 \sqrt{\tau}}\right)+\frac{1}{\sqrt{\tau \pi}}\left(\frac{1}{2} z^{2}-2 \tau\right) e^{-\frac{z^{2}}{4 \tau}}
$$

and $a^{\prime}$ and $b^{\prime}$ are given by (29).

For isothermal plates, the rate of heat transfer at the moving plate $\eta=0$ is given by

$$
\begin{aligned}
\theta^{\prime}(0) & \left.=\frac{\partial \theta}{\partial \eta}\right)_{\eta=0} \\
& = \begin{cases}\sum_{n=0}^{\infty}\left[\sqrt { \alpha } \left\{-H_{1}\left(a^{\prime} \sqrt{\alpha}, \tau\right)\right.\right. & \text { for } \alpha \neq 1, \\
\left.\left.+H_{1}\left(b^{\prime} \sqrt{\alpha}, \tau\right)\right\}\right] & \text { for } \alpha=1, \\
\sum_{n=0}^{\infty}\left[-H_{1}\left(a^{\prime}, \tau\right)+H_{1}\left(b^{\prime}, \tau\right)\right] & \end{cases}
\end{aligned}
$$

where $a^{\prime}$ and $b^{\prime}$ and $H_{1}(z, \tau)$ are given by (29) and (30).
Numerical results of the rate of heat transfer $-\theta^{\prime}(0)$ at the moving plate $(\eta=0)$ against the radiation parameter $R$ are presented in Table 1 and 2 for various values of Prandtl number $\operatorname{Pr}$ and time $\tau$. Table 1 shows that the rate of heat transfer $-\theta^{\prime}(0)$ increases with an increase in either radiation parameter $R$ or Prandtl number $P r$ for both ramped as well as isothermal wall temperature at the moving plate $\eta=0$. It is observed from Table 2 that the rate of heat transfer $-\theta^{\prime}(0)$ first increases and reaches maximum and then decreases with an increase in time $\tau$ while it increases with an increase in radiation parameter $R$ for ramped wall temperature. On the other hand, the rate of heat transfer $-\theta^{\prime}(0)$ increases with an increase in radiation parameter $R$ whereas it decreases with an increase in time $\tau$ for isothermal wall temperature. The negative value of $\theta^{\prime}(0)$ physically explains that there is heat flow from the plate.

Table 1. Rate of heat transfer $-\theta^{\prime}(0)$ at the moving plate $(\eta=0)$ when $\tau=1.1$

\begin{tabular}{|l|c|c|c|c|c|c|c|c|}
\hline & \multicolumn{5}{|c|}{ Ramped } & \multicolumn{4}{c|}{ Isothermal } \\
\hline$R \backslash P r$ & 4 & 5 & 6 & 7 & 4 & 5 & 6 & 7 \\
& & & & & & & & \\
\hline 0.5 & 0.00693 & 0.04923 & 0.12404 & 0.21940 & 0.56185 & 0.62817 & 0.68813 & 0.74326 \\
1.0 & 0.14922 & 0.31589 & 0.51755 & 0.73367 & 0.70432 & 0.78745 & 0.86261 & 0.93173 \\
1.5 & 0.30492 & 0.55519 & 0.82274 & 1.07976 & 0.78281 & 0.87521 & 0.95874 & 1.03556 \\
2.0 & 0.43403 & 0.73367 & 1.03004 & 1.29769 & 0.83336 & 0.93173 & 1.02066 & 1.10244 \\
\hline
\end{tabular}

Table 2. Rate of heat transfer $-\theta^{\prime}(0)$ at the moving plate $(\eta=0)$ when $\operatorname{Pr}=10$.

\begin{tabular}{|l|c|c|c|c|c|c|c|c|}
\hline & \multicolumn{5}{|c|}{ Ramped } & \multicolumn{3}{c|}{ Isothermal } \\
\hline$R \backslash \tau$ & 0.5 & 0.7 & 0.9 & 1.1 & 0.5 & 0.7 & 0.9 & 1.1 \\
\hline 0.5 & 1.25703 & 1.31834 & 1.27009 & 0.59548 & 1.31766 & 1.11363 & 0.98213 & 0.88837 \\
1.0 & 1.64645 & 1.90251 & 2.03880 & 1.33298 & 1.65178 & 1.39601 & 1.23116 & 1.11363 \\
1.5 & 1.83487 & 2.15527 & 2.38322 & 1.69267 & 1.83585 & 1.55158 & 1.36836 & 1.23766 \\
2.0 & 1.95412 & 2.30503 & 2.57805 & 1.89142 & 1.95441 & 1.65178 & 1.45673 & 1.31766 \\
\hline
\end{tabular}




\section{CONCLUSION}

Effect of radiation on unsteady Coutte flow between two vertical parallel plates with a ramped wall temperature has been investigated. It is found that the velocity decreases with increase in either radiation parameter $R$ or Prandtl number $\mathrm{Pr}$. An increase in Grashof number $\mathrm{Gr}$ leads to rise in the velocity $u_{1}$ of flow field. It is seen that the velocity $u_{1}$ increases with an increase in time $\tau$. It is also seen that the temperature $\theta$ decreases with increase in either radiation parameter $R$ or Prandtl number $\mathrm{Pr}$. The temperature increases with an increase in time. Further, it is observer that for fixed value of Grashof number $G r$, the shear stress $\tau_{x}$ decreases for both the cases of ramped and isothermal wall temperature with an increase in either radiation parameter $R$ or time $\tau$. The rate of heat transfer $-\theta^{\prime}(0)$ increases with an increase in either radiation parameter $R$ or Prandtl number $P r$ for both ramped as well as isothermal wall temperature at the moving plate $\eta=0$.

\section{REFERENCES}

[1] Schlichting, H. (1979). Boundary Layer Theory. McGrawHill, New York.

[2] Korycki, R.(2006). Sensitivity analysis and shape optimization for transient heat conduction with radiation. Int. J. Heat Mass Transfer. 49: 2033-2043.
[3] Gbaorun, F. K., Ikyo, B., Iyorzor, B. and Okanigbun, R. A.(2008). Heat model for temperature distribution in a laptop computer. J. of NAMP. 12: 201-206.

[4] Singh, A. K. (1988). Natural convection in unsteady Couette motion. Def. Sci. J. 38(1): 35-41.

[5] Ogulu, A. and Motsa, S. (2005). Radiative heat transfer to magnetohydrodynamic Couette flow with variable wall temperature. Physica Scripta. 71(4): 336-339.

[6] Mebine, P. (2007). Radiation effects on MHD Couette flow with heat transfer between two parallel plates. Global J. Pure and Appl.Math. 3(2): 1 - 12.

[7] Narahari, M. (2010). Effects of thermal radiation and free convection currents on the unsteady Couette flow between two vertical parallel plates with constant heat flux at one boundary. WSEAS Transactions on Heat and Mass Transfer. 5(1): 21-30.

[8] Deka, R. K. and Bhattacharya, A. (2011). Unsteady free convective Couette flow of heat generating/absorbing fluid in porous medium. Int. J. Math. Arch. 2(6): 853-863.

[9] Gbadeyan, J. A., Daniel, S. and Kefas, E. G. (2005). The radiation effect on electrohydrodynamic froth flow in vertical channel. J. Math. Associ. Nigeria. 32(2B): 388396.

[10] Chung, T. J.(2002). Computational Fluid Dynamics. Cambridge University Press. 